\title{
Transoral Excision of a Pedunculated Pleomorphic Sarcoma
}

\section{Blanca Alonso Martinez ${ }^{1 *}$ | Paula Martinez Pascual² | Ricardo Hitt Sabag³ | Juan Cantón de Seoane ${ }^{1}$}

*Correspondence: Blanca Alonso Martinez

Address: ${ }^{1}$ Internal Medicine Service. Severo Ochoa University Hospital, Spain; ${ }^{2}$ Otorhinolaryngology and Cervical-Facial Surgery Service. Severo Ochoa University Hospital, Spain; ${ }^{3}$ Medical Oncology Service. Severo Ochoa University Hospital, Spain e-mail $₫$ blancaalonsomartinez@hotmail.com

Received: 01 March 2021; Accepted: 03 March 2021

Copyright: (C) 2021 Alonso MB. This is an open-access article distributed under the terms of the Creative Commons Attribution License, which permits unrestricted use, distribution, and reproduction in any medium, provided that the original work is properly cited.

\section{ABSTRACT}

A 38-year-old man presented to the emergency department with a 2-month history of dysphagia and shortness of breath. He also reported a foreign body sensation in the throat. A pedunculated pleomorphic sarcoma was diagnosed and an oral excision was made.

Keywords: Transoral Excision, Pleomorphic Sarcoma, Pedunculated

\section{Clinical Image}

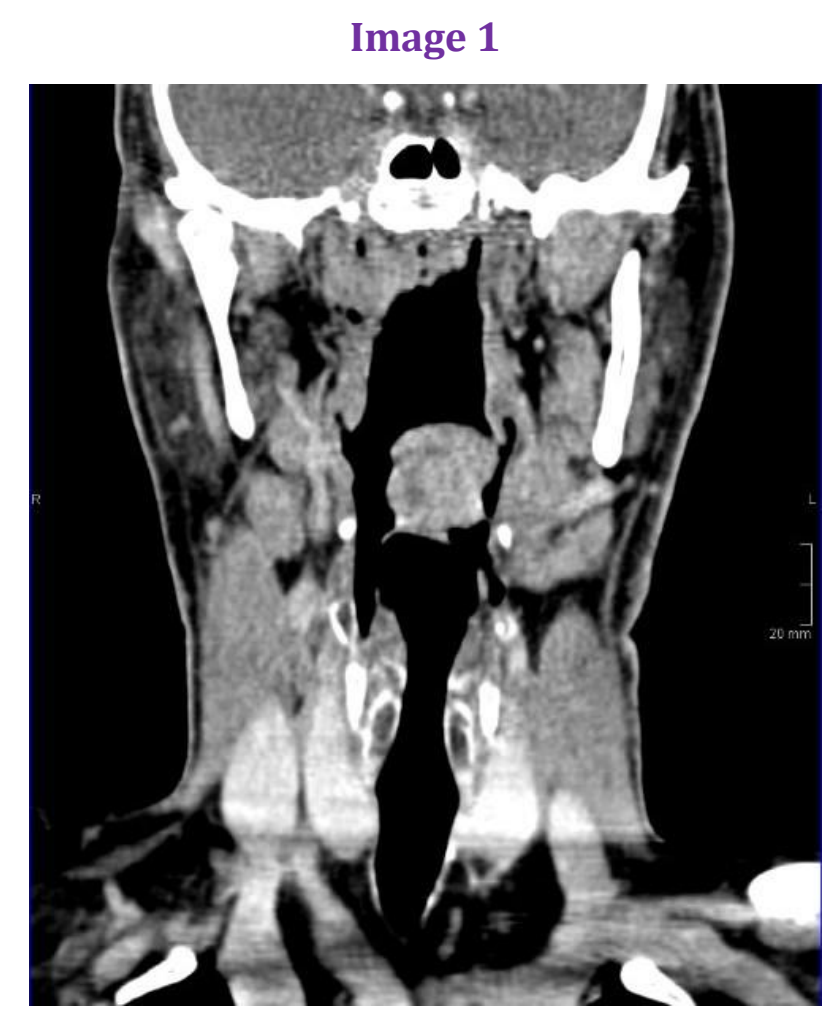


Image 2

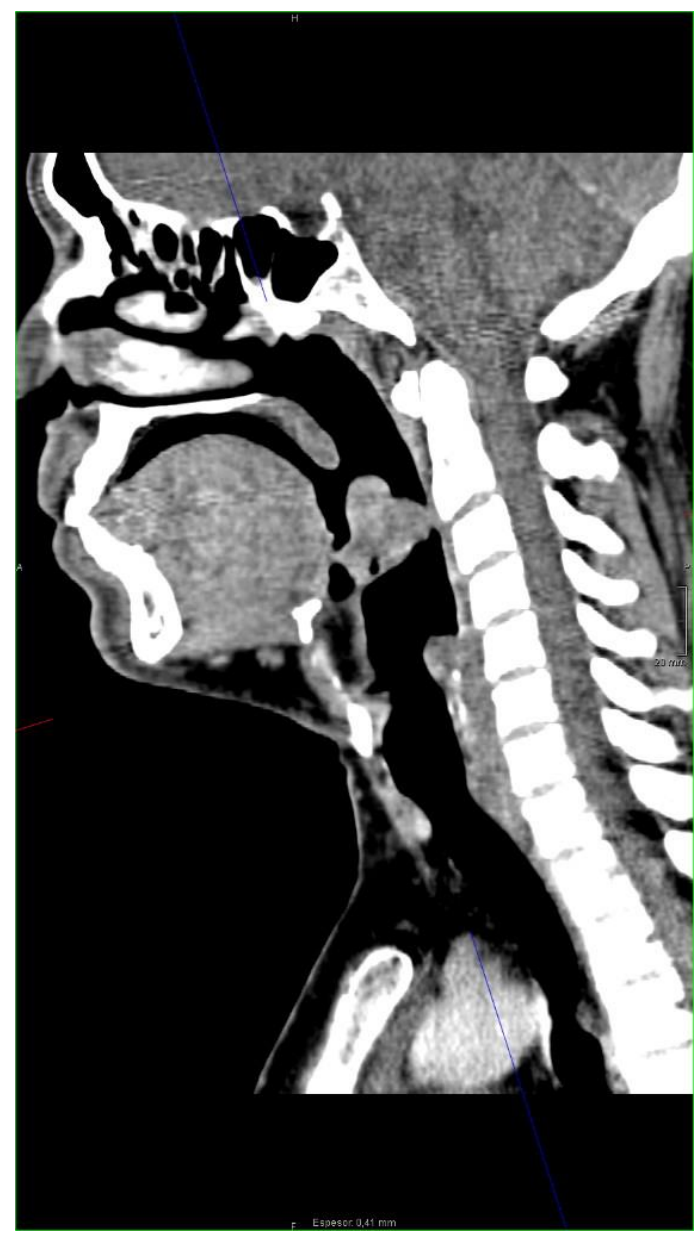

Image 3

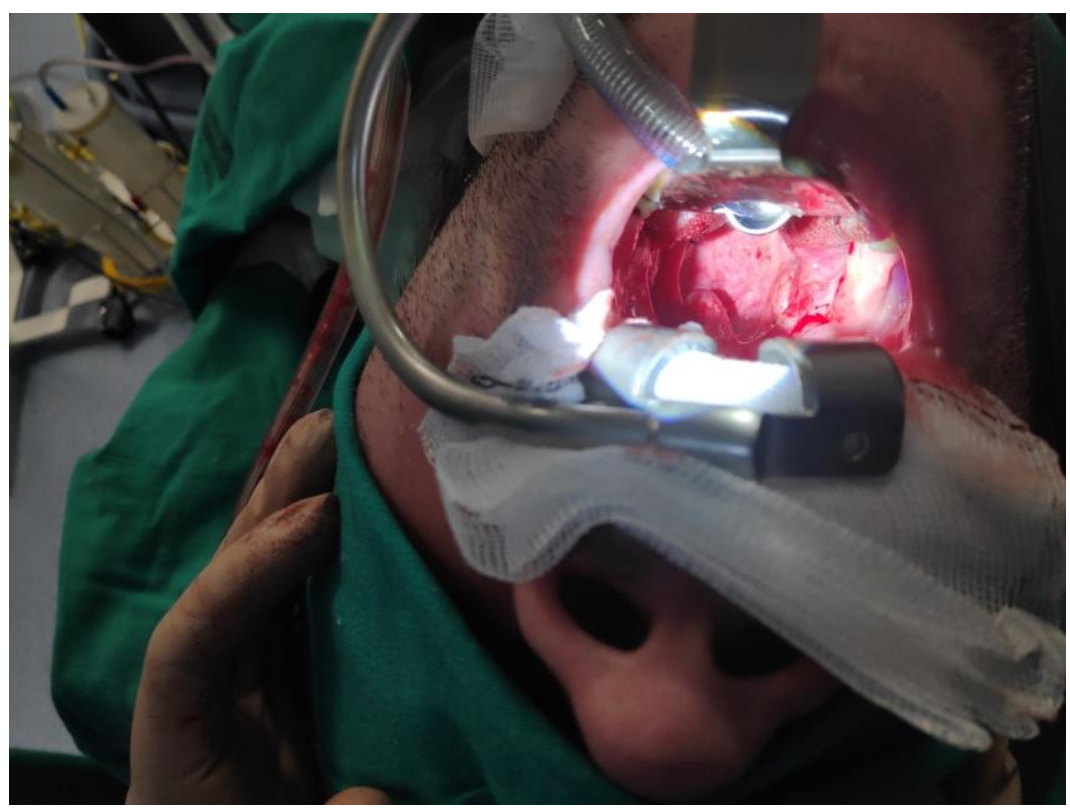


A 38-year-old man presented to the emergency department with a 2-month history of dysphagia and shortness of breath. He also reported a foreign body sensation in the throat.

The patient was evaluated by an Otolaryngologist, where $4 \mathrm{~cm}$ pedunculated mass of a smooth surface annexed to the lower portion of the left palatoglossus muscle was found in the oral cavity. A cervical CT sagittal section with intravenous contrast showed a solid, poly-lobulated, a clearly defined lesion of approximately $2 \times 3 \times 2.6 \mathrm{~cm}$ of anteroposterior, transverse and longitudinal axes respectively. It arose from the free edge of the epiglottis (Image 1 coronal section). The cervical nodes were within normal size and were not suggestive of malignancy (Image 2 sagittal section).

The lesion was excised by transoral surgery and the entire sample was sent for analysis. Pathological analysis of the mass revealed an undifferentiated pleomorphic sarcoma with free disease resection margins (Image 3). 